Università degli Studi di Milano

Facoltà di Scienze Matematiche, Fisiche e Naturali

Dipartimento di Informatica e Comunicazione

\title{
On Race Vulnerabilities in Web Applications
}

\author{
Roberto Paleari Davide Marrone Danilo Bruschi \\ Mattia Monga
}

DIMVA 2008 
Web applications

- many applications adopt the web paradigm: client-server model + HTTP protocol

- web servers are augmented with modules for the execution of server-side code

\section{Security issues}

- web applications are known to be subject to different attacks (e.g., SQLI, XSS, command injection)

- $\sim 60 \%$ of software vulnerabilities are specific to web applications 


\section{Web application framework}

\section{Single request}

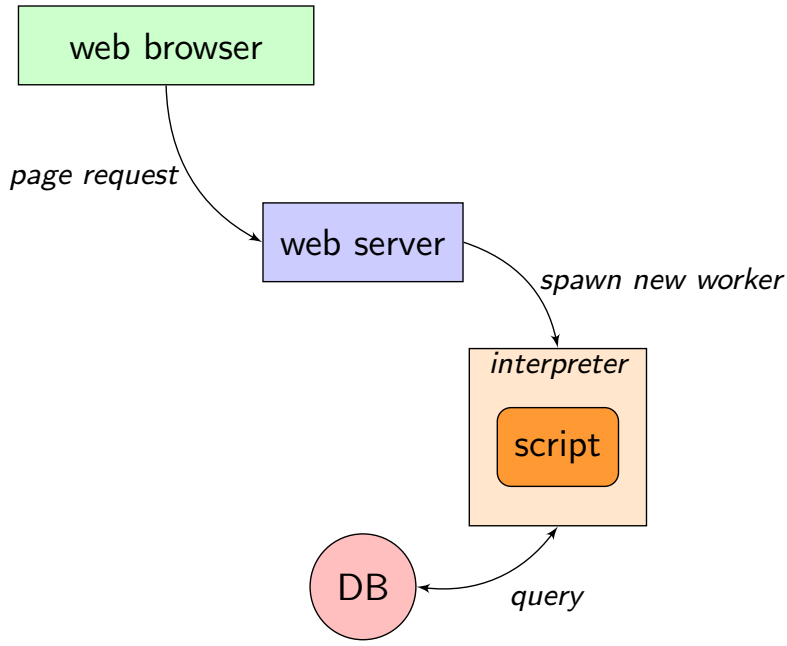




\section{Web application framework}

\section{Multiple parallel requests}

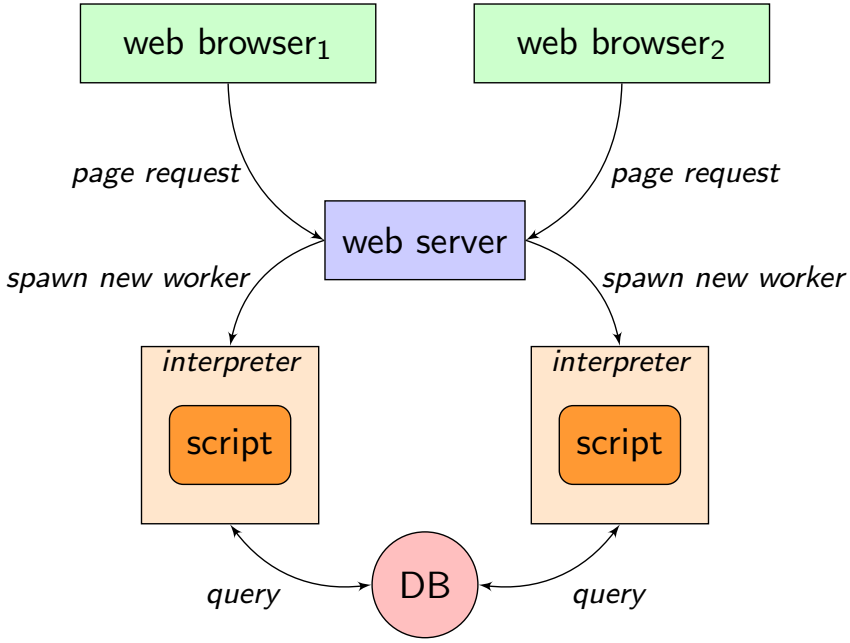




\section{Web application framework}

\section{Multiple parallel requests}

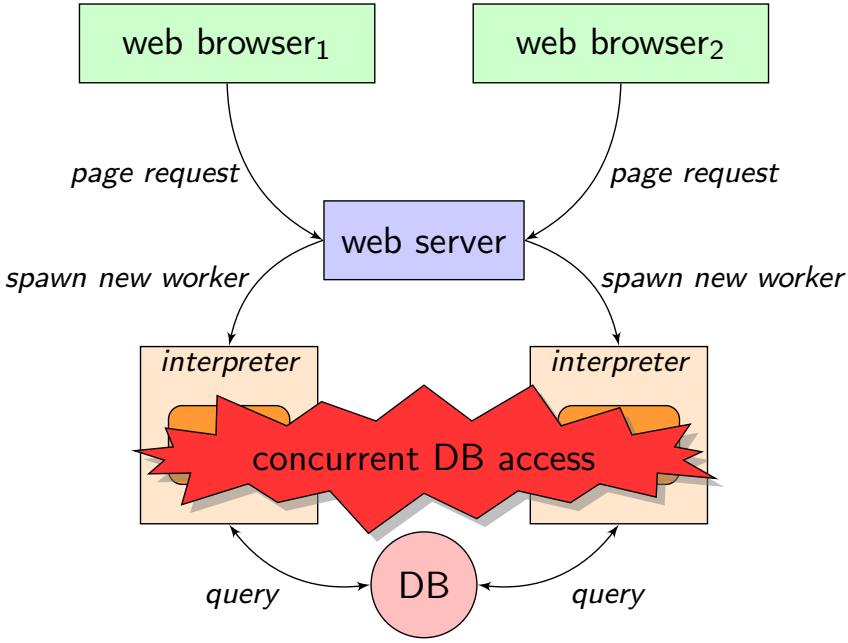




\section{Concurrency in web applications}

- web apps are made of different scripts that perform well-defined, sequential tasks

- scripts usually access some shared resources (e.g., a database)

- multiple script instances can be executed concurrently

- race conditions are well known, but their impact on web applications has not been investigated

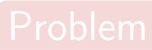

web programmers do not conceive their applications as

multi-threaded or multi-process entities

- unexpected parallelism can lead to unforeseen interactions

- parallelism can be controlled client-side

- synchronization primitives are seldom used, and their efficacy

is often system-dependent 


\section{Concurrency in web applications}

- web apps are made of different scripts that perform well-defined, sequential tasks

- scripts usually access some shared resources (e.g., a database)

- multiple script instances can be executed concurrently

- race conditions are well known, but their impact on web applications has not been investigated

\section{Problem}

web programmers do not conceive their applications as multi-threaded or multi-process entities

- unexpected parallelism can lead to unforeseen interactions

- parallelism can be controlled client-side

- synchronization primitives are seldom used, and their efficacy is often system-dependent 


\section{Motivating example}

1 \$res = mysql_query("SELECT credit FROM Users WHERE id=\$id");

2 \$row $=$ mysql_fetch_assoc (\$res);

3 if (\$row['credit'] >= 800) \{

4 <execute the requested operation>

5 \$new_credit $=$ \$row ['credit'] - 800;

6 \$res = mysql_query ("UPDATE Users SET credit=\$new_credit" . "WHERE id=\$id");

\}

\begin{tabular}{|c|c|c|c|}
\hline \multicolumn{2}{|c|}{$P_{1}$} & \multicolumn{2}{|c|}{$P_{2}$} \\
\hline Line & Data & Line & Data \\
\hline
\end{tabular}

\begin{tabular}{ll}
\hline \multicolumn{2}{c}{ Database } \\
\hline$I D$ & Credit \\
\hline \hline 50 & 2500 \\
\hline 92 & 820 \\
\hline $\mathbf{1 2 3}$ & $\mathbf{1 0 0 0}$ \\
\hline 205 & 1200 \\
\hline$\ldots$ & $\ldots$ \\
\hline
\end{tabular}




\section{Motivating example}

1 \$res = mysql_query("SELECT credit FROM Users WHERE id=\$id");

2 \$row $=$ mysql_fetch_assoc (\$res);

3 if (\$row['credit'] >= 800) \{

$4 \quad$ <execute the requested operation>

5 \$new_credit $=$ \$row ['credit'] - 800;

6 \$res = mysql_query ("UPDATE Users SET credit=\$new_credit" . "WHERE id=\$id");

\}

\begin{tabular}{cc|cc}
\hline \multicolumn{2}{c|}{$P_{1}$} & \multicolumn{2}{c}{$P_{2}$} \\
\hline Line & Data & Line & Data \\
\hline \hline 2 & (id: 123, credit: 1000$)$ & $\perp$ & $\perp$ \\
\hline
\end{tabular}

\begin{tabular}{ll}
\hline \multicolumn{2}{c}{ Database } \\
\hline$I D$ & Credit \\
\hline \hline 50 & 2500 \\
\hline 92 & 820 \\
\hline $\mathbf{1 2 3}$ & $\mathbf{1 0 0 0}$ \\
\hline 205 & 1200 \\
\hline$\ldots$ & $\ldots$ \\
\hline
\end{tabular}




\section{Motivating example}

1 \$res = mysql_query ("SELECT credit FROM Users WHERE id=\$id");

2 \$row $=$ mysql_fetch_assoc (\$res);

3 if (\$row['credit'] >= 800) \{

4 <execute the requested operation>

$5 \quad$ \$new_credit $=$ \$row ['credit'] - 800;

6 \$res = mysql_query ("UPDATE Users SET credit=\$new_credit" . "WHERE id=\$id");

\}

\begin{tabular}{cc|cc}
\hline \multicolumn{2}{c|}{$P_{1}$} & \multicolumn{2}{c}{$P_{2}$} \\
\hline Line & Data & Line & Data \\
\hline \hline 2 & (id: 123, credit: 1000) & $\perp$ & $\perp$ \\
\hline 4 & (id: 123, credit: 1000$)$ & 1 & $\perp$ \\
\hline
\end{tabular}

\begin{tabular}{ll}
\hline \multicolumn{2}{c}{ Database } \\
\hline$I D$ & Credit \\
\hline \hline 50 & 2500 \\
\hline 92 & 820 \\
\hline $\mathbf{1 2 3}$ & $\mathbf{1 0 0 0}$ \\
\hline 205 & 1200 \\
\hline$\ldots$ & $\ldots$ \\
\hline
\end{tabular}




\section{Motivating example}

1 \$res = mysql_query ("SELECT credit FROM Users WHERE id=\$id");

2 \$row $=$ mysql_fetch_assoc (\$res);

3 if (\$row['credit'] >= 800) \{

4 <execute the requested operation>

$5 \quad$ \$new_credit $=$ \$row ['credit'] - 800;

6 \$res = mysql_query ("UPDATE Users SET credit=\$new_credit" . "WHERE id=\$id");

\}

\begin{tabular}{cc|cc}
\hline \multicolumn{2}{c|}{$P_{1}$} & \multicolumn{2}{c}{$P_{2}$} \\
\hline Line & Data & Line & Data \\
\hline \hline 2 & (id: 123, credit: 1000) & $\perp$ & $\perp$ \\
\hline 4 & (id: 123, credit: 1000) & 1 & $\perp$ \\
\hline 4 & (id: 123, credit: 1000$)$ & 2 & (id: 123, credit: 1000$)$ \\
\hline
\end{tabular}

\begin{tabular}{ll}
\hline \multicolumn{2}{c}{ Database } \\
\hline$I D$ & Credit \\
\hline \hline 50 & 2500 \\
\hline 92 & 820 \\
\hline $\mathbf{1 2 3}$ & $\mathbf{1 0 0 0}$ \\
\hline 205 & 1200 \\
\hline$\ldots$ & $\ldots$ \\
\hline
\end{tabular}




\section{Motivating example}

1 \$res = mysql_query ("SELECT credit FROM Users WHERE id=\$id");

2 \$row $=$ mysql_fetch_assoc (\$res);

3 if (\$row['credit'] >= 800) \{

4 <execute the requested operation>

$5 \quad$ \$new_credit $=$ \$row ['credit'] - 800;

6 \$res = mysql_query ("UPDATE Users SET credit=\$new_credit" . "WHERE id=\$id");

\}

\begin{tabular}{cc|cc}
\hline \multicolumn{2}{c|}{$P_{1}$} & \multicolumn{2}{c}{$P_{2}$} \\
\hline Line & Data & Line & Data \\
\hline \hline 2 & (id: 123, credit: 1000$)$ & $\perp$ & $\perp$ \\
\hline 4 & (id: 123, credit: 1000$)$ & 1 & $\perp$ \\
\hline 4 & (id: 123, credit: 1000$)$ & 2 & (id: 123, credit: 1000$)$ \\
\hline 5 & (id: 123, credit: 1000$)$ & 4 & (id: 123, credit: 1000$)$ \\
\hline
\end{tabular}

\begin{tabular}{ll}
\hline \multicolumn{2}{c}{ Database } \\
\hline$I D$ & Credit \\
\hline \hline 50 & 2500 \\
\hline 92 & 820 \\
\hline $\mathbf{1 2 3}$ & $\mathbf{1 0 0 0}$ \\
\hline 205 & 1200 \\
\hline$\ldots$ & $\ldots$ \\
\hline
\end{tabular}




\section{Motivating example}

1 \$res = mysql_query ("SELECT credit FROM Users WHERE id=\$id");

2 \$row $=$ mysql_fetch_assoc (\$res);

3 if (\$row['credit'] >= 800) \{

4 <execute the requested operation>

$5 \quad$ \$new_credit $=$ \$row ['credit'] - 800;

6 \$res = mysql_query ("UPDATE Users SET credit=\$new_credit" . "WHERE id=\$id");

\}

\begin{tabular}{cc|cc}
\hline \multicolumn{2}{c|}{$P_{1}$} & \multicolumn{2}{c}{$P_{2}$} \\
\hline Line & Data & Line & Data \\
\hline \hline 2 & (id: 123, credit: 1000$)$ & $\perp$ & $\perp$ \\
\hline 4 & (id: 123, credit: 1000$)$ & 1 & $\perp$ \\
\hline 4 & (id: 123, credit: 1000$)$ & 2 & (id: 123, credit: 1000$)$ \\
\hline 5 & (id: 123, credit: 1000$)$ & 4 & (id: 123, credit: 1000$)$ \\
\hline
\end{tabular}

\begin{tabular}{ll}
\hline \multicolumn{2}{c}{ Database } \\
\hline$I D$ & Credit \\
\hline \hline 50 & 2500 \\
\hline 92 & 820 \\
\hline 123 & 200 \\
\hline 205 & 1200 \\
\hline$\ldots$ & $\ldots$ \\
\hline
\end{tabular}




\section{Motivating example}

1 \$res = mysql_query ("SELECT credit FROM Users WHERE id=\$id");

2 \$row $=$ mysql_fetch_assoc (\$res);

3 if (\$row['credit'] >= 800) \{

4 <execute the requested operation>

$5 \quad$ \$new_credit $=$ \$row ['credit'] - 800;

6 \$res = mysql_query ("UPDATE Users SET credit=\$new_credit" . "WHERE id=\$id");

\}

\begin{tabular}{cc|cc}
\hline \multicolumn{2}{c|}{$P_{1}$} & \multicolumn{2}{c}{$P_{2}$} \\
\hline Line & Data & Line & Data \\
\hline \hline 2 & (id: 123, credit: 1000$)$ & $\perp$ & $\perp$ \\
\hline 4 & (id: 123, credit: 1000$)$ & 1 & $\perp$ \\
\hline 4 & (id: 123, credit: 1000$)$ & 2 & (id: 123, credit: 1000$)$ \\
\hline 5 & (id: 123, credit: 1000$)$ & 4 & (id: 123, credit: 1000$)$ \\
\hline
\end{tabular}

\begin{tabular}{ll}
\hline \multicolumn{2}{c}{ Database } \\
\hline$I D$ & Credit \\
\hline \hline 50 & 2500 \\
\hline 92 & 820 \\
\hline $\mathbf{1 2 3}$ & 200 \\
\hline 205 & 1200 \\
\hline$\ldots$ & $\ldots$ \\
\hline
\end{tabular}




\section{Race conditions in web applications}

\section{Case studies}

- tested several open-source apps

- 2 real-world closed-source SMS applications, both found to be vulnerable ( . . without having access to their source code!)

${ }^{a}$ Text messages for mobile phones. 


\section{Race conditions in web applications}

\section{Case studies}

- tested several open-source apps

- 2 real-world closed-source $\mathrm{SMS}^{a}$ applications, both found to be vulnerable (... without having access to their source code!)

${ }^{a}$ Text messages for mobile phones.

How to dynamically spot race conditions?

- we focus on LAMP applications

- interactions between multiple instances of the same script

- limited to races on database accesses 


\section{How to spot race conditions?}

\section{Framework overview}

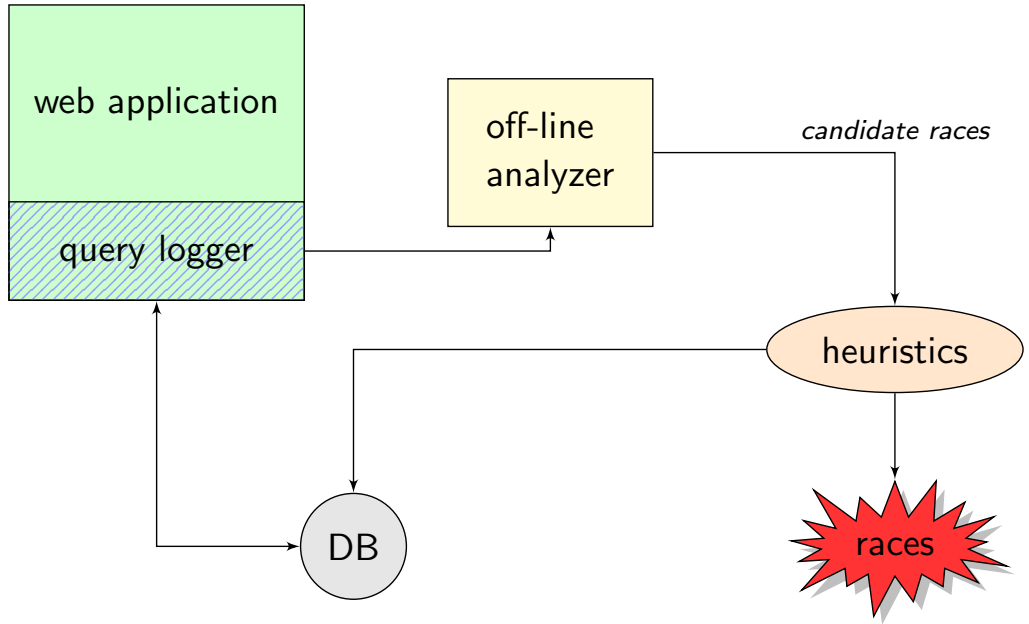




\section{Off-line analysis}

\section{Candidate race conditions}

Idea

- monitor application's SQL queries

- interdependent queries could lead to race conditions

\section{Off-line analysis algorithm}

- logged queries: $Q=\left\langle q_{1}, q_{2}, \ldots, q_{n}\right\rangle$

- $\forall q \in Q$ compute schema objects in use $(q)$ and $\operatorname{def}(q)$

- candidate races are $\left(q_{i}, q_{j}\right) \in Q^{2}: i<j \wedge$ use $\left(q_{i}\right) \cap \operatorname{def}\left(q_{j}\right) \neq \emptyset$

A simple example:

Query 1

SELECT id, credit FROM Users

WHERE id $=123$;
Query/2

UPDATE Users

SET credit $=100$

WHERE id $=123$; $\square=$ used

$\square=$ defined 


\section{Off-line analysis}

\section{Heuristics: WHERE clauses}

disjoint WHERE clauses can lead to false positives:

Query 1

SELECT id

FROM Sessions

WHERE expiry_time <= 123;
Query.

DELETE

FROM Sessions

WHERE expiry_time > 123;

\section{Solutions}

- dynamically query the DB for a conjunction of WHERE clauses $(\rightarrow$ efficient, but not sound)

- constraint solver ( $\rightarrow$ sound, but expensive and does not support all SQL constructs) 


\section{Off-line analysis}

More heuristics

\section{Attribute-relation bindings}

- it is not always apparent to which relation an attribute belongs (e.g., SELECT $a_{1}, a_{2}$ FROM $T_{1}, T_{2}$ )

- $\Rightarrow$ actively query the application database

\section{Annotations}

- synchronization attempts can lead to false positives

- $\Rightarrow$ annotations to avoid reporting a race between a pair of SQL queries 


\section{Evaluation}

\begin{tabular}{|l||l|r|r|c|}
\hline \multicolumn{1}{|c||}{ Application } & Category & Queries & FP & \multicolumn{1}{c|}{ TP } \\
\hline Joomla! 1.5RC4 & CMS & 4086 & 0 & $55(2)$ \\
\hline phpBB 3.0.0 & forum & 2236 & 0 & $35(4)$ \\
\hline WordPress 2.3.2 & blog/CMS & 3638 & 0 & $47(4)$ \\
\hline Zen Cart 1.3.8a & shopping cart & 35194 & 0 & $46(1)$ \\
\hline
\end{tabular}

\section{What kind of vulnerabilities did we find?}

Highly application-dependent. Some examples:

- bypass brute force checks

- vote multiple times with parallel vote requests

- circumvent anti-flooding features 


\section{Limitations}

\section{Dynamic, database-level analysis}

- analysis is completely dynamic

- we only take into account database queries

\section{Lack of support for synchronization primitives}

Why?

- during evaluation, we found really few synchronization attempts!

- lack of a set of "standard" synchronization primitives

- future work ;-) 


\section{Conclusions}

\section{Contributions}

- study of the impact of race conditions on web applications

- novel detection technique

- working experimental prototype for LAMP applications

\section{Future work}

- consider interactions between different scripts

- employ program analysis techniques to extract more information about the application's logic

- take into account synchronization primitives 


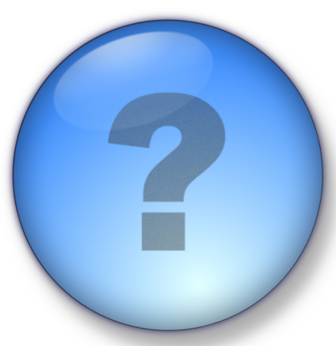

Questions? 